\title{
Strukturen Sehen. Über die Karriere eines Hexagons in der quantitativen Revolution
}

\author{
Boris Michel \\ Universität Erlangen-Nürnberg, Institut für Geographie, Wetterkreuz 15, 91058 Erlangen, Germany \\ Correspondence to: Boris Michel (boris.michel@fau.de)
}

Received: 4 May 2016 - Revised: 27 September 2016 - Accepted: 3 October 2016 - Published: 9 November 2016

Kurzfassung. Publications from the early quantitative revolution in geography saw a significant change in the use of visual material. While the old regionalist paradigm of "Länderkunde" was dominated by images of "geographical individuals" the new geography was dominated by abstract models and visualized laws and theories. Overall visual material gains in importance, both quantitatively and qualitatively. This paper follows the changed functions, possibilities and promises of visualizing epistemic things in geography's new paradigm. This is done by following the translations, transformations and mobilizations of the famous hexagon Walter Christaller published in his 1933 "Theory of central places in South Germany". Since the 1940s this Hexagon has become not only an icon of the new geography, but an instrument for making quantitative-theoretical thinking in the geography plausible and at the same time to build a visual bridge between the old and the new geography.

\section{Einleitung}

Dieser Beitrag nahm seinen Ausgangspunkt bei einigen marginalen Kritzeleien, die nicht für die Publikation bestimmt waren. In einem Fall begegneten sie in Form geometrischer Figuren auf Transparentpapier und Schmierzetteln in einem archivierten Nachlass und in einem anderen Fall in Form von Vandalismus an Bibliothekseigentum. Im Zentrum dieser Kritzeleien standen Christallers Theorie der zentralen Orte und dessen Visualisierung als Hexagon. Sie betrafen aber auch Weiterführungen durch Christaller und andere Geographen ${ }^{1}$. Diese Kritzeleien deuteten in unterschiedlicher Weise darauf hin, dass Praktiken der Visualisierung für die quantitativ-theoretische Geographie der 1950er und 1960er Jahre eine Bedeutung gewannen, die sie in dieser Form für die länderkundliche Geographie nicht besaßen. Sie sind Teil eines Denkens mit den Augen, das sich unterscheidet von der Art und Weise wie in der klassischen Geographie gesehen und visualisiert wurde.

\footnotetext{
${ }^{1}$ In der Tat sind unter den zitierten Texten der quantitativtheoretischen Geographie, auf die in diesem Artikel Bezug genommen wird, keine Geographinnen, so dass mit Bezug auf die 1950er und 1960er Jahre ausschließlich die männliche Form verwendet wird.
}

Eingebunden sind dieser Beitrag sowie das Interesse an jenen Kritzeleien in ein disziplingeschichtliches Projekt, welches sich mit der Rolle von Visualität für die Geographie des 20. Jahrhunderts befasst. Dieses Projekt unternimmt eine Präzisierung und Differenzierung der Behauptung, die Geographie sei eine ,visuelle“ Wissenschaft (Rose, 2003). Dabei geht es um die Frage, wie in historisch unterschiedlicher Weise Fragen des Blicks, der Visualisierung und der Sichtbarkeit in der Geographie verhandelt und praktiziert wurden (Michel, 2013, 2015). Dazu gehören Diskussionen um die Rolle ästhetischer Erfahrung bei Autoren wie Ratzel, Feldforschungspraktiken und die Schulung des „Geographischen Blicks", die Übersetzung von Welt in Bilder, Karten oder Skizzen oder die Beschreibung der Geographie als die Wissenschaft der im Raume sichtbaren Dinge (Gregory, 1994; Michel, 2014; Henniges, 2014). Dies sind Momente, die gemeinhin mit der „Klassischen Geographie“ assoziiert werden, also einer idiographischen, individualisierenden und holistischen Perspektive. Der Großteil der Forschung zur Geschichte von Geographie und Visualität arbeitet sich daher an der Geographie zwischen der Visualisierungspraxis Humboldts (Daston, 2010; Schneider, 2012; Godlewska, 1999) und dem Blick auf die Landschaft als räumlichem Individuum und Superorganismus in der Kul- 
turgeographie Sauers (Rose, 1996; Jackson, 1989; Cosgrove, 1984) ab. Die quantitativ-theoretische Geographie und deren Visualisierunsgpraktiken und Visualisierungstheorien sind bisher nur in begrenztem Umfang in den Blick wissenschaftsgeschichtlicher Untersuchungen gerückt (Barnes, 1998; Michel, 2016a; Gregory, 1994:52 ff.). Deren Spezifik steht im Zentrum der Fragestellung dieses Beitrags.

Die quantitativ-theoretische Geographie begriff sich als radikaler Gegenentwurf zur länderkundlichen und regionalgeographischen Perspektive. Im Folgenden interessiert daher die Frage, wie sich diese Neuformulierung auf die Rolle von Sichtbarkeit und Visualität in der Formierungsphase der quantitativ-theoretischen Geographie auswirkte. Dabei geht es nicht nur darum, dass sich mit der quantitativen Revolution die dominanten Darstellungsformen geändert haben und andere Gegenstände visualisiert werden, sondern um die These, dass die Geographie der quantitativen Revolution in anderer Weise auf Visualisierung angewiesen war.

Im Zentrum dieses Artikels steht die wohl bekannteste Darstellung der quantitativ-theoretischen Geographie, das Hexagon Christallers und dessen Karriere seit den 1930er Jahren. Das Hexagon aus Christallers „,Die zentralen Orte in Süddeutschland" (Christaller, 1933a) wurde insbesondere seit den 1950er Jahren zu einer ikonischen Visualisierung der neuen Geographie und diente Autoren wie Bunge oder Haggett als Beleg für die Tragfähigkeit dieses neuen Denkens (Bunge, 1962; Haggett, 1965). Zudem verdichten sich in ihm paradigmatisch die wesentlichen Momente quantitativ-theoretischer Visualisierung in der Geographie. Christiallers Hexagon dient diesem Aufsatz als Ausgangspunkt, der Karriere dieser Darstellung in den unterschiedlichsten Formen und Kontexten zu folgen. Seit den frühen 1940er Jahren taucht es vielfach in wissenschaftlichen Publikationen, in Planungsempfehlungen, als Logo, in Metaphern und eben in Kritzeleien auf. Dabei finden sich direkte Reproduktionen ebenso wie stark veränderte Transformationen, die beanspruchen, Christallers Modell empirisch oder theoretisch zu konkretisieren, zu übersetzen, zu erweitern oder auch zu überwinden.

Für diesen Aufsatz interessieren weniger die Gestaltung oder ästhetischen Qualitäten dieser Visualisierungen. Der Beitrag ist keine bildwissenschaftliche Untersuchung. Vielmehr geht es um die Produktivität dieser recht simplen und technisch wenig elaborierten Abbildung in der frühen quantitativen Revolution. Damit verbunden ist die These, dass diese Produktivität des Hexagons für die Etablierung und Stabilisierung quantitativ-theoretischen Denkens in der Geographie gerade in einer Unschärfe und Hybridität dieser diagrammatischen Darstellung lag, welche dessen objektivistischen und szientistischen Anspruch überschritt. Der Erfolg des Hexagons, so die hier vertretene These, resultierte gerade daraus, dass es nicht das strenge und klare Abbild des theoretischen und deduktiv gewonnenen Modells des Systems der zentralen Orte war, als welches es beschrieben wurde. Vielmehr wird deutlich, dass es im Sinne von Latour offen für vielfäl- tige Übersetzungen, Transformationen und Mobilisierungen blieb. Es vermittelte zwischen deduktivem und induktivem Zugang zwischen relativen und absoluten Raumbegriffen, es macht das Neue intelligibel durch seine Vermittlung zum Alten.

Im folgenden Abschnitt wird zunächst kurz der konzeptionelle Rahmen dargelegt, an dem sich dieses Projekt orientiert. Daran anschließend werden in zwei Abschnitten allgemeine Momente geographischer Visualisierung der quantitativ-theoretischen Geographie herausgearbeitet. Diese Skizze bildet den Hintergrund, vor dem die Karriere des besagten Hexagons bei Christaller sowie der frühen quantitativ-theoretischen Geographie nachgezeichnet wird. Deutlich wird dabei sowohl dessen paradigmatischer Charakter für die Visualisierung der quantitativ-theoretischen Geographie wie auch dessen Besonderheit, die es so attraktiv als Ikone eines neuen geographischen Denkens machte.

\section{Wissenschaftsgeschichtliche Verortung}

Der Hintergrund dieser Arbeit ist eine wissenschaftsgeschichtliche Perspektive, die weder Ideengeschichte großer Ideen sein möchte, noch eine externalistische Soziologie von Wissenschaft. Stattdessen interessiert sie diese Perspektive für die alltäglichen Praktiken und Arrangements, die in den gereinigten Texten wissenschaftlicher Publikationen nicht mehr sichtbar sind (Hagner, 2001; Daston, 2009; Rheinberger, 2010). Damit rückt der Blick auf die vielfältigen Aufschreibesysteme, die Welt in Wissen übersetzen und durch diese Übersetzung nutzbar und produktiv machen (Latour, 1990). Hierzu zählen Fotos, Skizzen, Diagramme und natürlich Karten auf der einen Seite und Instrumente der Registrierung, Erzeugung und Segmentierung auf der anderen Seite. Diese sind allesamt mehr als nur Illustrationen und unwesentliche Hilfsmittel, sondern wirkungsvolle Beteiligte wissenschaftlicher Praxis (Daston und Galison, 2007; Rheinberger, 2009). Diese Praxis ist zumeist geprägt von Bemühungen der Reinigung, der Behauptung von Eindeutigkeit und der Herstellung von Widerspruchsfreiheit. Was aber die historische und ethnographische Wissenschaftsforschung immer wieder deutlich gemacht hat, sind die messiness, Situiertheit und Alltäglichkeit wissenschaftlichen Arbeitens, eine messiness, die in Kritzeleien sichtbarer ist, als in einem veröffentlichten Text, die aber auf beides angewiesen ist.

Wenn sich Wissenschaftsgeschichte und Science Studies seit den 1980er Jahren mit diesen Verfahren der Übersetzung, Transformation und Mobilisierung intensiv beschäftigt haben und auch dort eine visuelle Wende konstatiert wurde (Burri und Dumit, 2008), so werden auch in der Geographie Fragen der Visualität und der Wissenschaftsforschung breit diskutiert. Ausgehend von Arbeiten feministischer Wissenschaftskritik (Haraway, 1988) waren es in den 1990er Jahren insbesondere feministische Arbeiten, die eine Kritik geographischer Beobachtung und Visualität formuliert ha- 
ben (Rose, 1993). Auch in der deutschsprachigen Geographie ist in jüngerer Zeit ein wachsendes Interesse an Science Studies und der Rolle von Visuellem zu beobachten (Schlottmann und Miggelbrink, 2009, 2015; Michel, 2013; Wintzer, 2014). Darüber hinaus ist gerade die wissenschaftsgeschichtliche Perspektive in der Geographie weiterhin unterbelichtet. Zwar formulierte Rose ihre Kritik am geographischen Blick vor dem Hintergrund einer Auseinandersetzung mit Sauer und schreibt Livingstone in seinem Aufsatz „In defence of situated messiness" ein frühes Plädoyer für eine geographische Wissenschaftsgeschichte in der hier formulierten Perspektive (Livingstone, 1992), insgesamt bleibt das Feld einer Wissenschaftsgeschichte geographischer Visualisierungspraktiken aber überschaubar (Livingstone, 1992; Driver, 1995, 2013; Barnes, 1998; Michel, 2013; Dyce, 2013; MacDonald und Withers, 2015).

\section{Quantitatives zur Visualisierungspraxis der quantitativen Revolution}

Obgleich das Besondere an den genannten Kritzeleien ist, dass sie nicht zur Publikation und dem Gebrauch von anderen vorgesehen wurden, sind sie Ausdruck einer allgemeineren Beobachtung. In Publikationen aus den frühen Jahren der quantitativen Revolution (bis etwa Mitte der 1960er Jahre) fällt die Vielzahl von Abbildungen, Karten, Skizzen, Graphen und anderen Formen visuellen Materials auf. Dies zeigt sich beispielsweise an drei der einflussreichsten frühen methodologischen Beiträgen. Isards „Location and SpaceEconomy“, die erste umfangreiche Arbeit zu einer neuen quantitativ-theoretischen regional science, hat sechzig Abbildungen auf dreihundert Seiten. Diese reichen von ,,a hypothetical region (Isard, 1956:4) oder ,,input proportions and equilibrium for an agricultural enterprise" (ebd., 192) bis zur „division of a market region between two sources of each of two raw materials" (ebd., 261). Auf den zweihundert Seiten von Bunges „Theoretical Geograhy“ finden sich über einhundert Diagramme, Tabellen und Karten vom ,Shifting of U.S. Highway 99 in the Tacoma-Seattle region in Washington“ (Bunge, 1962:30), ,A map with azimuthal logarithmic distance scale“ (ebd., 54) oder „Predicted shifts in medical service regions“ (ebd., 113). Haggett veröffentlichte „Locational Analysis in Human Geography“ (Haggett, 1965) mit über zweihundert Abbildungen. Ähnliches lässt sich auch für eine Vielzahl von Büchern und Publikationen in jenen Zeitschriften konstatieren, die bereits früh quantitativ-theoretische Arbeiten publizierten.

Bemerkenswert ist dabei, dass diese starke Zuhilfenahme von visuellem Material ganz besonders für solche Texte gilt, die als theoretische Grundlegungsversuche einer neuen Geographie angelegt sind. Es sind also gerade theoretische Argumente, die einer visuellen Unterstützung bedürfen. Das ist eine Neuerung gegenüber der klassischen Geographie der Länderkunde, die in ihren empirischen Arbeiten durchaus auf
Visualisierungen angewiesen war, in theoretischen Beiträgen auf diese aber kaum zurückgriff.

Manche dieser Abbildungen wie Bunges Darstellung von Straßen in der Region um Seattle, die er dem Mäandern eines Flusslaufs gegenüberstellt und sozialphysikalisch diskutiert, haben einen konkreten realräumlichen Bezugspunkt. Andere wie Isards ,hypothetical region“ dienen als rein abstrakte Modelle räumlicher Beziehungen. Die Zunahme visuellen Materials ist dabei kein Ausdruck technischen Fortschritts, der die Reproduktionskosten von Abbildungen gesenkt hat oder eines allgemeinen Bedeutungsgewinns visueller Kommunikation in modernen Gesellschaften, sondern Ausdruck eines veränderten wissenschaftlichen Paradigmas - auch wenn dieses natürlich nicht losgelöst von diesen sozialen und technologischen Kontexten begriffen werden darf. $^{2}$

Dies überrascht aus zwei Gründen. Zum einen waren methodologische Bücher der Geographie vor dieser Zeit weitgehend frei von Bildern als Hilfsmittel um Aussagen eine visuelle Evidenz an die Seite zu stellen. Hettners „Die Geographie“, ein Buch, dessen Anspruch es war, das Lebenswerk Hettners zu verdichten, enthält keine Abbildung, auch wenn Hettner sich darin ausführlich mit Karten, Photographie und Beobachtung beschäftigt. ${ }^{3}$ Hartshornes ,The Nature of Geography“, das englischsprachige und wissenschaftstheoretisch elaboriertere Pendent zu Hetterns Arbeit, enthält lediglich eine Abbildung. Bei dieser handelt es sich um ein Diagramm, das die Beziehung der Geographie zu den anderen Wissenschaften illustriert und damit keine ,geo“Visualisierung ist (Hartshorne, 1939:147). Ähnliches gilt für den ersten Band von Ratzels „Anthropo-Geographie“, auch wenn es im zweiten Band einige Tabellen und Karten gibt (Ratzel, 1882, 1891). Empirische Arbeiten des späten 19. und frühen 20. Jahrhundert hingegen waren oft reich an Photographien, Tabellen, Karten und Zeichnungen.

Ein anderer Punkt ist jedoch für diesen Text bedeutsamer. So überrascht es auf den ersten Blick, dass gerade eine Geographie so stark auf Visualisierung angewiesen ist, die in ihrer konzeptionellen Anlage in solchem Maße auf Abstraktion, Mathematik und Raumgesetze fokussiert und die sich so stark gegen die Idee richtet, die Geographie sei eine Disziplin der schlichten Beschreibung der Verteilung der sichtbaren Dinge auf dem Gesicht der Erde (Schaefer, 1953:227). Bevor sich dem Hexagon zugewandt wird, soll daher in aller Kürze versucht werden, die geographische Visualisierungspraxis der quantitativen Revolution zu skizzieren.

\footnotetext{
${ }^{2}$ Für die Plausibilität dieser Annahme spricht auch, dass länderkundliche oder kulturgeographische Arbeiten dieser Zeit nicht eine vergleichbare Zunahme sehen.

${ }^{3}$ Lediglich eine visuelle Metaphorik bezüglich bildlicher Schilderung fällt auf (Hettner, 1927) - sein berühmtes ,Schema“ (Hettner, 1919:20) hat Hettner selbst in keiner Publikation visualisiert, die auch heute noch allgegenwärtige Darstellung geht auf einen Beitrag von Ernst Weigt zurück (Weigt, 1957:35).
} 
4

\section{Qualitatives zur Visualisierungspraxis der quantitativen Revolution}

Mit der quantitativ-theoretischen Wende verlor die Geographie ihren sichtbaren Gegenstand, der ihr als idiographischer und positivistischer Wissenschaft noch unproblematisch und ganz nichtmetaphorisch vor Augen erschien. Für die Geographie als quantitativ-theoretische Raumwissenschaft, der es um Modelle und Raumgesetzte ging, waren die Dinge der länderkundlichen Geographie kein wissenschaftlicher Gegenstand mehr. Deshalb gab es in deren bildlichen Schilderungen, in illustrativen Zeichnungen und Photographien nichts zu sehen. Entscheidend ist für die geographische Betrachtung und Visualisierung nun nicht mehr ,das Erscheinungsbild“, sondern „,ihre Funktion“ (Christaller, 1933a:22). Im Zentrum stehen nun die Beziehungen der Dinge und nicht das Aussehen der Dinge selbst (Haggett, 1965:2). Aber gerade deshalb war diese neue Geographie so sehr auf die Erzeugung von Visuellem angewiesen, auf neue Formen der wissenschaftlichen Bildgebung und visuellen Argumentation. Mit der quantitativ-theoretischen Geographie setzten sich Diagramme, Visualisierungen von Raummustern und Modellen geographischer Gesetze als wesentliche Formen geographischer Visualisierung durch. Die Bilder dieser Geographie sind nicht nur abstrakter und erscheinen einfacher, sondern mit ihnen gehen ganz andere Vorstellungen von der Funktion von Visualisierung für wissenschaftliche Erkenntnisproduktion einher. In ihnen zeigt sich die neue Fokussierung auf Fragen der Ordnung, Symmetrie und des Gleichgewichts ebenso wie eine Betonung eines relativen Raumbegriffs, ein Anschluss an Modelle und Metaphern aus Physik und Ökonomie und eine Ausrichtung auf Steuerung und Planung. Dabei werden Visualisierungen, die zuvor in erster Linie als Beweis von Augenzeugenschaft fungierten, machtvoller, da sie nun für die Visualisierung von Theorien und die Sichtbarmachung von abstrakten Raumgesetzen stehen. Die Wirkmächtigkeit dieser abstrakten Visualisierungen geht damit über die Bezeugung des Einzelfalles hinaus (Michel, 2016b). Um diesen Bedeutungsgewinn zu erklären, erscheinen mir vier Argumente besonders zentral:

1. Die visuelle Tradition: Geographie hat in Forschung und Lehre eine lange visuelle Tradition (Rose, 2003; Tuan, 1979). Wenn die Geographie traditionell eine starke Affinität zu Visualität aufweist, so helfen Visualisierungen dabei, das neue Paradigma in diese Tradition einzuschreiben. Dass sich die nun theoretisch und methodologisch radikal reformulierte Geographie auf Verfahren der Bildgebung ihrer epistemischen Dinge stützt, mag dazu beigetragen haben, die epistemische Gemeinschaft der Geographie in einer Zeit zu stabilisieren, die vielfach als epistemologischer Bruch wahrgenommen wurde. Wenn also die Art der Visualisierung ebenso einer Modernisierung unterzogen wurde wie das, was als visualisierungswürdig erachtet wurde, so wurde ein
Vertrauen auf die Evidenzproduktion der Visualisierung nicht in Frage gestellt. Versuche, räumliche Gesetze direkt in Form mathematischer Formeln zu beschreiben, blieben zunächst rar.

2. Ein visuelles Paradigma: Zugleich gibt es eine Spezifik des Visuellen der quantitativ-theoretischen Geographie. Einerseits verliert der Gegenstand der Geographie an unmittelbarer Sichtbarkeit. Statt der Beschreibung, Klassifikation und Abbildung von einzigartigen Objekten der sichtbaren Landschaft interessieren die quantitativ-theoretische Geographie nun Funktionen, Netzwerke und Strukturen. Um vertrauensvolles Wissen zu produzieren, ist sie auf Techniken der Sichtbarmachung angewiesen. Andererseits ist die theoretische Grundlegung der neuen Geographie in hohem Maße mit Fragen der Visualisierung verbunden. Mit Schaefers Aufforderung, Geographie müsse die Wissenschaft räumlicher Muster werden (Schaefer, 1953), wird die Geographie zu einer Wissenschaft der angewandten Geometrie. Die quantitativ-theoretische Wende ist also nicht so sehr eine Wende zu Mathematik per se, sondern zu einer Mathematik, die in besonderem Maße visuell ist. Die Geographie der quantitativ-theoretischen Wende war also in doppelter Hinsicht besonders stark visuell.

3. Unterstützung von anderswo: Ähnlich wie Metaphern können Bilder helfen eine Analogie zwischen dem Bekannten und dem Unbekannten herzustellen. Anders als in der ausgesprochen solipsistischen Geographie zuvor, bezogen sich Geographen der frühen quantitativen Revolution vielfach auf Modelle, Theorien und Visualisierungen anderer Disziplinen. Sie fand ihre neuen Verbündeten in erster Linie in Physik und Wirtschaftswissenschaften. Die quantitativ-theoretische Geographie kennt eine Vielzahl visueller Darstellungen von Brechungsgesetzen, Mustern von Potenzialen oder Gravitationskräften (Stewart, 1947; Warntz, 1957a, b). Zudem werden Analogien zwischen Mustern und Gesetzen hergestellt. So ist zu lesen, dass es für die neue Perspektive der Geographie letztlich keine Rolle spiele, ob Wasser oder Menschen sich in Netzwerken bewegen, es um die Verteilung von Menschen oder Kartoffeln ginge oder das Phänomen Gletscher oder religiöse Bewegungen beträfe (Bunge, 1962, 1966a:208). Die dahinterliegenden Raumgesetze seien die gleichen.

4. Stabilisierung im Moment des Neuen: Etwa zeitgleich mit der quantitativ-theoretischen Wende in der Geographie hat Kuhn hervorgehoben, dass neue wissenschaftliche Paradigmen stabilisiert und mit Autorität versehen werden müssen, damit diesen vertraut wird (Kuhn, 1962). Visualisierung und Regulierung des Blicks sind anerkannte Verfahren, ein solches Vertrauen zu produzieren. Wenn Bilder gerade im Moment der Sichtbar- 
machung von Neuem so wichtig sind, dann ist es kaum überraschend, dass Harveys „Explanation in Geography“ (Harvey, 1969), das sowohl der Meilen- wie Grabstein der quantitativ-theoretischen Geographie wurde, kaum mehr auf Visualisierung angewiesen war. Zwar finden sich dort rund 30 Abbildungen, etwa Diagramme der ,The ,Baconian“ route to scientific explanation“, diese sind aber gerade keine "Geo"Visualisierungen. Zugleich konnte Harvey machtvolle Verbündete aus der modernen Wissenschaftsphilosophie mobilisieren.

Diese Beobachtungen der qualitativ und quantitativ veränderten Dimensionen der Nutzung, von Visualisierungen in den Publikationen der frühen quantitativ-theoretischen Geographie bilden den Hintergrund, vor dem im Folgenden der Karriere von Christallers Hexagon nachgegangen wird.

\section{Das Hexagon als Ikone eines neuen geographischen Denkens und Handelns.}

Eine beständig wiederkehrende Abbildung in Texten der quantitativ-theoretischen Wende ist Christallers Hexagon zur Darstellung der Ergänzungsgebiete im System der zentralen Orte (Abb. 1). Gezeigt wird dort die verschränkte Hexagonalgeometrie, die sich aus den unterschiedlichen Zentralitätsgraden von Funktionen in hierarchischen Systemen ergibt. Die Darstellung bildet die von Christaller proklamierten räumlichen Gesetzmäßigkeiten ab und zeigt, dass hinter der scheinbar willkürlichen Verteilung von Siedlungen im Raum ein ,ordnendes Prinzip“ (Christaller, 1933a:11) zu finden ist. Neben dieser Abbildung finden sich im Text noch eine Reihe weiterer Darstellungen, die eine hexagonale räumliche Anordnung von Funktionen und Räumen plausibilisieren sollen. Die drei von Christaller hervorgehobenen räumlichen Strukturierungsprinzipien, das Versorgungsprinzip, das Verkehrsprinzip und das Absonderungsprinzip werden im Kapitel „Beziehungen der Statik“, visuell belegt, wohingegen die „Vorgänge der Dynamik“ und das, was Christaller als die dynamische Betrachtungsweise beschreibt, ohne Visualisierung auskommen.

Christallers „Die zentralen Orte in Süddeutschland. Eine ökonomisch-geographische Untersuchung über die Gesetzmäßigkeit der Verbreitung und Entwicklung der Siedlungen mit städtischen Funktionen" wurde ab den 1940er Jahren zu einer der wichtigsten Referenzen einer neuen Geographie. In diesem Text wurde die klarste Formulierung eines neuen geographischen Denkens erblickt. Zahlreiche frühe Arbeiten der anglophonen quantitativ-theoretischen Geographie verweisen als entscheidende Vorarbeit oder Inspiration auf diese Arbeit. Seit den 1960er Jahren wurde in der Anglophonen Geographie auf Christaller als den ,father of theoretical geography“ (Bunge, 1962) Bezug genommen - wohingegen er in der deutschsprachigen Geographie weiterhin als angewandter Geograph galt (für eine aktuelle Rekonstruktion der Rezeption siehe: Kegler, 2015, 2016). In seiner Christaller

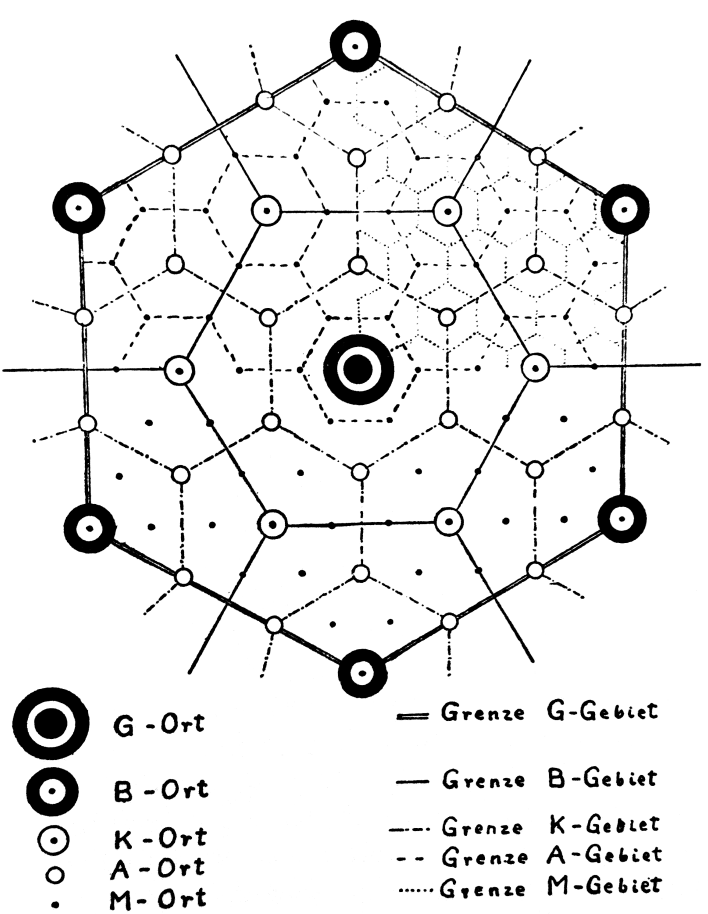

Abb. 1. Christaller (1933:71).

gewidmeten „Theoretical Geography“ schreibt Bunge, ,,[if]it were not for the existence of central place theory, it would not be possible to be so emphatic about the existence of a theoretical geography independent of any set of mother sciences“" (Bunge, 1962:129).

So wie Christallers Arbeit als Entwurf einer theorie- und hypothesen-geleiteten Geographie gelesen wurde, so drückt seine Visualisierung eine visuelle Sprache aus, die paradigmatisch ist für die szientistische Wende in der Geographie: Geometrie, Abstraktion und Distanziertheit heben Christallers Arbeit visuell und sprachlich aus der Geographie seiner Zeit heraus und bilden eine wesentliche Vorlage für eine neue Form geographischer Visualisierung. Dabei profitiert diese Visualisierung gewiss davon, dass sie Anschluss herstellt an die zeitgenössischen Formen wissenschaftlicher Bildgebung.

Angesichts dieser paradigmatischen Form sowie der unzähligen Bezüge darauf überrascht es, dass das Hexagon zwar ein immer wiederkehrender Topos der Geographie geworden ist, es aber fast keine wissenschaftsgeschichtliche Auseinandersetzung mit diesem stattfand (als Ausnahme: Barnes, 1998). Dies überrascht umso mehr, als die Bedeutung des Hexagons als visuellem Instrument von frühen Adepten deutlich herausgestellt wurde.

In seiner Laudatio anlässlich der Verleihung einer Auszeichnung des schwedischen Königs an Christaller 1967 konstatiert Hägerstrand, dass es kaum möglich sei, Christallers theoretischen Reflektionen bezüglich Größe, Zahl und geographischer Verteilung zentraler Orte „nur durch das Wort und ohne die Hilfe graphischer Illustrationen“" 
(Hägerstrand, 1967) zu verstehen. Für Hägerstrand ist es ausgemacht, dass Christallers Ideen in einer solchen Weise radikal für die Geographie sind, dass sie zum einen visuell übersetzt werden mussten, um für die bisherige Geographie Sinn zu machen. Zum anderen steht für Hägerstrand fest, dass Christaller eine neue Art des visuellen Denkens (und nicht nur eine neue Art des Visualisierens) in die Geographie einführt. Er sei der erste gewesen, der in der Geographie ,seine Gedanken durch mathematische Linien ausdrückte“ (Hägerstrand, 1967). Um Christaller zu verstehen, sei es nötig dieses Hexagon zu sehen, auch weil es im Feld mit dem altgeographischen Blick, der in der Landschaft die Zeit und die Beziehungen der Dinge untereinander liest, nicht sichtbar ist. Vielmehr musste Christaller für seine Arbeit erst Telefonanschlüsse zählen, um hieraus ein hierarchisches Netzwerk zentraler Orte abzuleiten, eine für die Geographie dieser Zeit ungewöhnlich abstrakte und indirekte Methode der Produktion wissenschaftlicher Tatsachen (Christaller, 1933a). Die Bildgebung realisiert hier erst den Gegenstand vom dem gesprochen wird. Trotz der langen kartographischen Tradition war dies neu für die Geographie, nicht aber für andere Disziplinen.

Das, was zur visuellen Ikone der neuen Geographie werden sollte, wurde veröffentlicht, deutlich bevor es so etwas wie eine quantitativ-theoretische Wende in der Geographie gab. Es wurde veröffentlicht von jemandem, der aus der Ökonomie zur Geographie kam und dessen Innovation für die Geographie zu erheblichen Teilen in der Übersetzung wirtschaftswissenschaftlicher Rationalitäten in die Geographie lag - seien es Deduktion, die Vorstellung eines idealen und homogenen Raums oder der homo oeconomicus als Akteur. Christaller, der seine Arbeit als ,gleichzeitig geographische und ökonomische Forschung" (Christaller, 1941b:117) bezeichnet, hatte bei Weber studiert und wollte seine Dissertation zunächst nicht in der Geographie einreichen. Für die Wirtschaftswissenschaften mit einer langen Tradition der diagrammatischen Visualisierung ökonomischer Sachverhalte (Buck-Morss, 1995) und deren räumlichen Ordnung etwa durch von Thünen oder Weber waren Visualisierungen abstrakter Strukturen weniger provokativ, als für die Geographie.

Betrachtet man die Diskussionen in der Geographie bis in die 1950er Jahre - und auch in Hägerstrands Laudatio deutet sich dies an -, so wird das Provokative dieser Darstellungen deutlich. So diskutieren Geographen, ob die Hexagone im Feld oder auf der Karte sichtbar seien oder nicht und was deren empirischer oder epistemischer Status sei. Am Hexagon kristallisieren sich Fragen bezüglich des Status von Modell und Theorie, Empirie und Beobachtung und damit Fragen zum Kern und den Grenzen der Geographie (Tschierske, 1961:92; Bobek, 1935; Smailes, 1946:87 f.; Weigt, 1951:262). Die Hexagone, so Gould in Retrospektive, mögen für eine ältere Generation von Geographen schwierig zu sehen gewesen sein, aber für jene, die in der Theorie der zentralen Orte ausgebildet und im neuen geographischen
Blick geschult wurden, böten sie einen neuen Blick auf praktische Probleme (Gould, 1979:141).

Selbst wenn man Hägerstrands Annahme, dass es unmöglich sei, den ,ziemlich komplizierten geometrischen Charakter" (Hägerstrand, 1967) von Christallers Theorie ohne deren Visualisierung zu begreifen, für eine Übertreibung im Rahmen einer Laudatio hält, und Christaller auch nicht der erste war, der in der Geographie theoretische Konzepte durch Visualisierung materialisierte, so wird klar, dass diese Darstellung und die damit verbundenen Metaphern bedeutsam und kontrovers waren. Das Hexagon der zentralen Orte wurde Ausgangspunkt theoretischer und empirischer Weiterführungen und zum Inbegriff idealer und harmonischer Raumstrukturen. Es wurde zu einer visuellen Leitmetapher der neuen Geographie. Dabei resultieren Erfolg und Produktivität gerade daraus, dass dieses eben nicht ein strenges und klares Abbild des theoretisch und deduktiv gewonnenen Modells des Systems der zentralen Orte ist. Vielmehr war es offen für die Übersetzung, Transformation und Mobilisierung. Wie in den nächsten Kapiteln deutlich werden wird, zeigt sich dies in der Arbeit Christallers selbst wie auch bei späteren Autoren, die diese aufgriffen.

\section{1 Übersetzungen 1}

Das Hexagon, so wie es Christaller als „rein deduktiv“ (Christaller, 1933a:15) gewonnenes Modell optimaler Raumstrukturen präsentierte, ließ sich transformieren und praktisch wenden. Theorie und Modell beschreiben nicht nur ein theoretisches Optimum in einem räumlichen Vakuum. Vielmehr - und das ist neu für die Geographie - konnte das Modell Grundlage dazu werden, eine auf dieser Basis als nichtoptimal bzw. defizitär beschriebene räumliche Struktur zu optimieren. Dies wird besonders deutlich an Christallers vieldiskutierter Arbeit während des Nationalsozialismus (Kegler, 2015; Rössler, 1990; Barnes, 2015). Christaller formuliert aber auch schon 1933 und noch vor seiner beruflichen Einbindung in den NS-Staat „Grundsätzliches zu einer Neugliederung des Deutschen Reiches und seiner Verwaltungsbezirke". In dem so betitelten Aufsatz formulierte er Überlegungen zu einer neuen Raumordnung auf der Basis rationaler Raumgesetze und damit zu einer Überführung von Theorie in Planung und Praxis (Christaller, 1933b).

Neben dieser Nutzbarmachung für die Transformation eines durch Krieg und völkischen Rassismus als leer und homogen vorgestellten und hergestellten Raum (quasi als Raum ohne Volk) konnte das Modell auch konzeptionell an politische Vorgaben und Aushandlungsprozesse zwischen technokratischer und völkisch-organizistischer Ausrichtung nationalsozialistischer Raumordnungspolitik angepasst werden. Es war also praktisch wie auch diskursiv für Transformationen offen. Wie Rössler schreibt, wurde Christallers Standortlehre in der Reichsarbeitsgemeinschaft für Raumforschung der Vorwurf gemacht, Ausdruck einer liberalistischen Weltanschauung zu sein (Rössler, 1990:147) und seine explizi- 
te Bezugnahme auf einen homo oeconomicus, legt diese Interpretation nahe (Christaller, 1941b:121). Wenn Christaller 1933 und zum Teil auch später eine recht moderne Sprache verwendet, Metaphern aus der Physik und Geometrie wählte um seine Ideen zu plausibilisieren, so sind es in den frühen 1940er Jahren ,,bienenwabenförmige, sehr organisch wirkende Struktur[en] der Siedlungslandschaft" (ebd., 133), die Christaller hier sieht, zeichnet und realisieren möchte. Entsprechend sind nicht nur die kartographische Darstellung der direkten Planung und die darin visualisierten Forderungen nach „Entwickeln“ oder „Abwerten“ von Orten auf ,typische Größe“" wesentlich weniger einem streng geometrischen Muster unterworfen (Abb. 2). Auch die konzeptionellen Überlegungen sind nun in einer weit organischeren Weise visualisiert und konzeptualisiert (Abb. 3).

Abweichungen von der „Bienenwabenstruktur“ gelten Christaller nun als Indikator für die Degeneration einer völkischen Gemeinschaft und nicht mehr als zu erklärende ökonomische oder physisch-geographische Abweichungen. Die modernistische Perspektive wird landromantisch gewendet. So schreibt er: „Die Planung hat hier eine schöne und große Aufgabe vor sich, in diesem Sinne die Landschaft zu ordnen, sie damit zu gesunden und zu stärken, wobei das im Liberalismus verdorbene und überwucherte Netz der zentralen Orte wieder hergestellt oder neugebildet wird" (Christaller, 1942:55). Anders sieht es aus, wenn Raumentwicklung gegen die Harmonie hexagonaler Raumgesetze betrieben wird. In ,Amerika hingegen, wo das Verkehrsprinzip bei der Verteilung der zentralen Orte vorherrschend gewesen ist, findet man eine mehr dem Quadrat angenäherte Struktur [...] sie wirkt künstlich und gemeinschaftshemmend" (Christaller, 1941b:133). Was zunächst als wirtschaftsgeographisches Argument die Raumgesetze einer Siedlungsstruktur von Akteuren beschreiben sollte, die als homo oeconomicus handeln, wird nun übersetzt in ein völkisches und geopolitisches Argument.

Insgesamt bleiben Christallers Formulierungen flexibel bzw. widersprüchlich zwischen modernistischer Raumtheorie und einer Anpassung an die völkisch-organische Seite des Nationalsozialismus - so wie auch der Nationalsozialismus selbst immer eine reaktionär-modernistische und eine antimodernistische Seite hatte (Herf, 1984). Seinen im Sinne einer quantitativ-theoretischen Geographie modernsten Text veröffentlicht Christaller 1941 unter dem Titel ,Raumtheorie und Raumordnung“ im Archiv für Wirtschaftsplanung (Christaller, 1941b). Im Anschluss an ein Plädoyer für eine deduktive Konstruktion abstrakter Raumgesetze schreibt Christaller bezüglich der Rolle visueller Darstellung: ,Jeder Vorgang im Raume ist geometrisch meßbar und darstellbar. Es sind diese geometrischen Darstellungen nicht bloße Symbole, Verdeutlichungen eines Vorgangs oder einer Erscheinung im Raume, sondern die grundsätzlich richtigen Raumbeziehungen des Vorgangs“ (ebd., 121).

Das Hexagon hat für Christaller dabei nicht nur illustrierende, sondern auch erkenntnisgenerierende Funktion und dies widerspricht seiner Behauptung, die Theorie der zentralen Orte sei als ,rein deduktiv“ zu verstehen. In seinem autobiographischen Text ,Wie ich zu der Theorie der zentralen Orte gekommen bin“" schreibt Christaller über sein Arbeiten an der Theorie der zentralen Orte: ,ich verband Städte gleicher Größe durch gerade Linien, einmal, um festzustellen, ob im Eisenbahn- oder Straßennetz gewisse Regeln erkennbar seien, ..., zum andern, um die Abstände zwischen gleich großen Städten zu messen. Dabei füllten sich die Karten mit Dreiecken, oft gleichseitigen Dreiecken ... die sich zu Sechsecken zusammenschlossen“ (Christaller, 1968:95 f.). Im Nachlass von Christaller finden sich eine Vielzahl von Skizzen und Zeichnungen - darunter auch die eingangs erwähnten Kritzeleien in denen Hexagone sowohl zu analytischen Überlegungen wie auch zu Anlage einer neuen Raumordnung Europas (Abb. 4). Zudem begegnen in Berichten, Briefen und autobiographischen Texten vielfältige Anekdoten über das Zeichnen von Hexagonen und anderen Geometrien in Atlanten, Reiseführern und auf Karten, beispielsweise in den im Nachlass Christallers versammelten Briefen, die Ruth Hottes von Geographen im Rahmen ihrer Arbeit zum Leben Christallers erhielt (NL Christaller, Institut für Länderkunde Leipzig, 577). Es gibt also eine Produktivität nicht nur als deduziertes Model, sondern auch eine der alltäglichen Praxis. Das Verbinden von Punkten produziert Neues, da es Muster vor Augen führt. Es ist ein Denken mit den Augen, das für die Geographie in dieser Form neu ist. Natürlich haben auch länderkundliche Geographen skizziert und gekritzelt. Die Nachlässe älterer Geographen sind reich an solchen, diese sind aber keine theoretischen Modelle, sondern entweder im Modus der „Naturtreue“ gezeichnete Charakterbilder geographischer Klassen oder einzelne Raumindividuen.

Für Christaller standen Hexagone im Zentrum seiner Arbeit, seine Theorie war um wirkmächtig zu werden auf diese Visualisierung angewiesen. Die Visualisierung war flexibel, sie konnte sowohl zum Verstehen von Mustern und Prozessen als auch zur Transformation von Raum und Gesellschaft benutzt werden. Hier stehen zu bleiben, hieße aber, sich an einer Person aufzuhängen, die, wie Barnes schreibt, es souverän schaffte, immer zur falschen Zeit am falschen Ort zu sein (Barnes, 1998:102). Ein Sozialist und Stadtreformer in den 1920er Jahren und wichtiger Protagonist nationalsozialistischen Raumplanung, der nach dem Krieg zu den wenigen in der Geographie gehörte, denen ihre NS Vergangenheit vorgeworfen wurde und der dann zu seiner Ehrung durch die American Geographical Society nicht reisen durfte, da ihm als KPD-Funktionär ein Visum für die USA verwehrt bliebt. Dennoch, so Barnes weiter, ,his famous diagram emerged in Anglo-American economic geography at precisely the right place and the right time“ (ebd., 102). Dieser richtige Zeitpunkt war eine Krise der Disziplin und die Etablierung einer neuen quantitativ- und theoretisch ausgerichteten Geographie. 


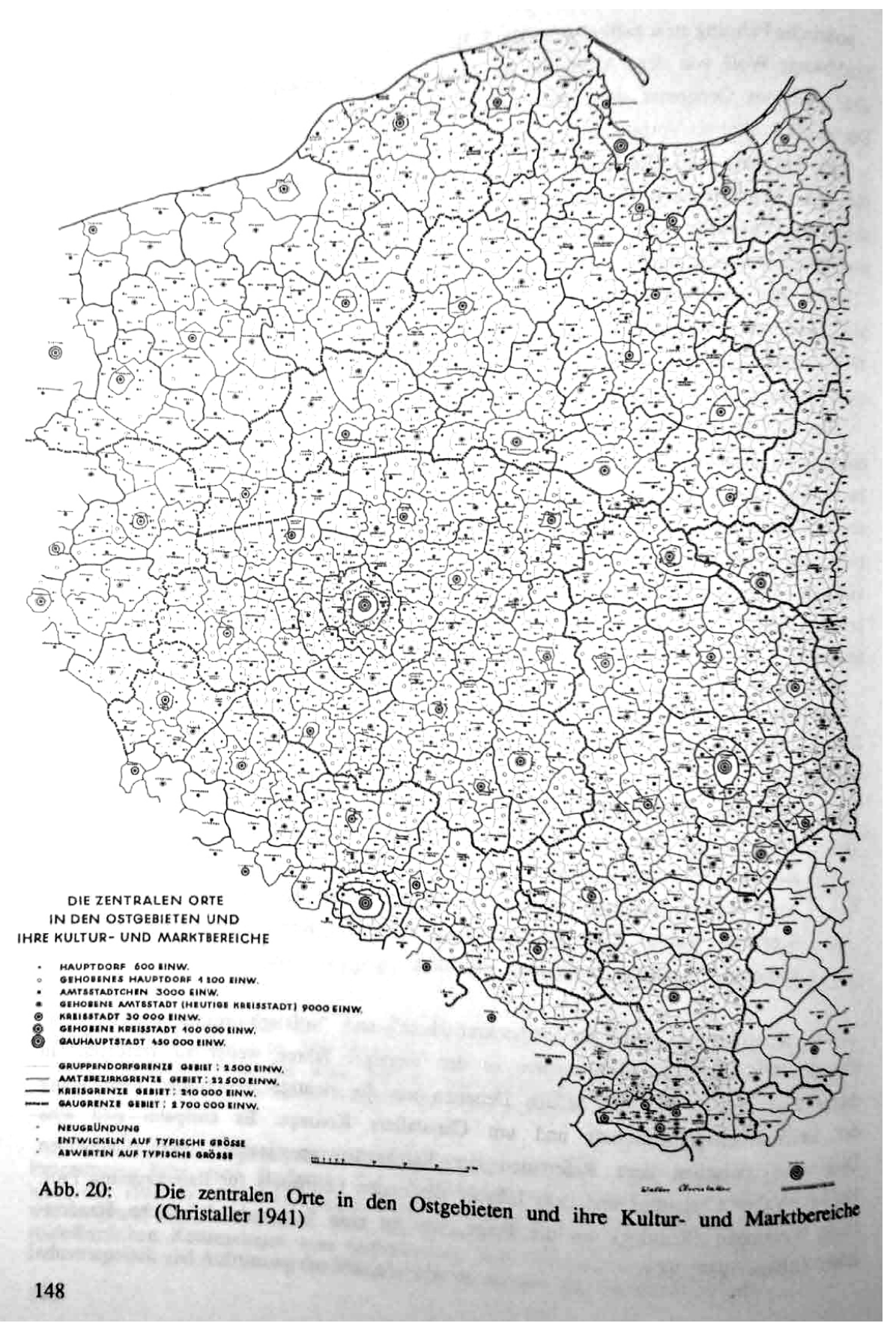

Abb. 2. Kartenbeilage (Christaller, 1941a).

\section{2 Übersetzungen 2}

Christaller, die Theorie der zentralen Orte und eben dieses Hexagon wurden ab den 1940er Jahren nicht nur für die staatliche Raumplanung in sehr unterschiedlichen nationalen und politischen Kontexten wichtig (Trezib, 2014; Kegler, 2015), sondern auch zu einem der wesentlichen Verbündeten der quantitativen Revolution in der anglophonen Geographie. Gerade hierin deutet sich eine Produktivität an, die über die konkrete Arbeit Christallers und die Reichweite und Plausibilität der dort formulierten Aussagen hinausgeht (von Böventer, 1969).

Unterstützt wurde dies von den deutlich stärker mathematisch hergeleiteten und theoretisch solideren Arbeiten von Lösch. Mit seinem Text „The Nature of Economical Regions“ (Lösch, 1938) war Lösch zudem früher auf Englisch rezipierbar als Christaller. Löschs Arbeit galt dabei vielfach als mathematische Ausarbeitung und theoretische Präzisierung 


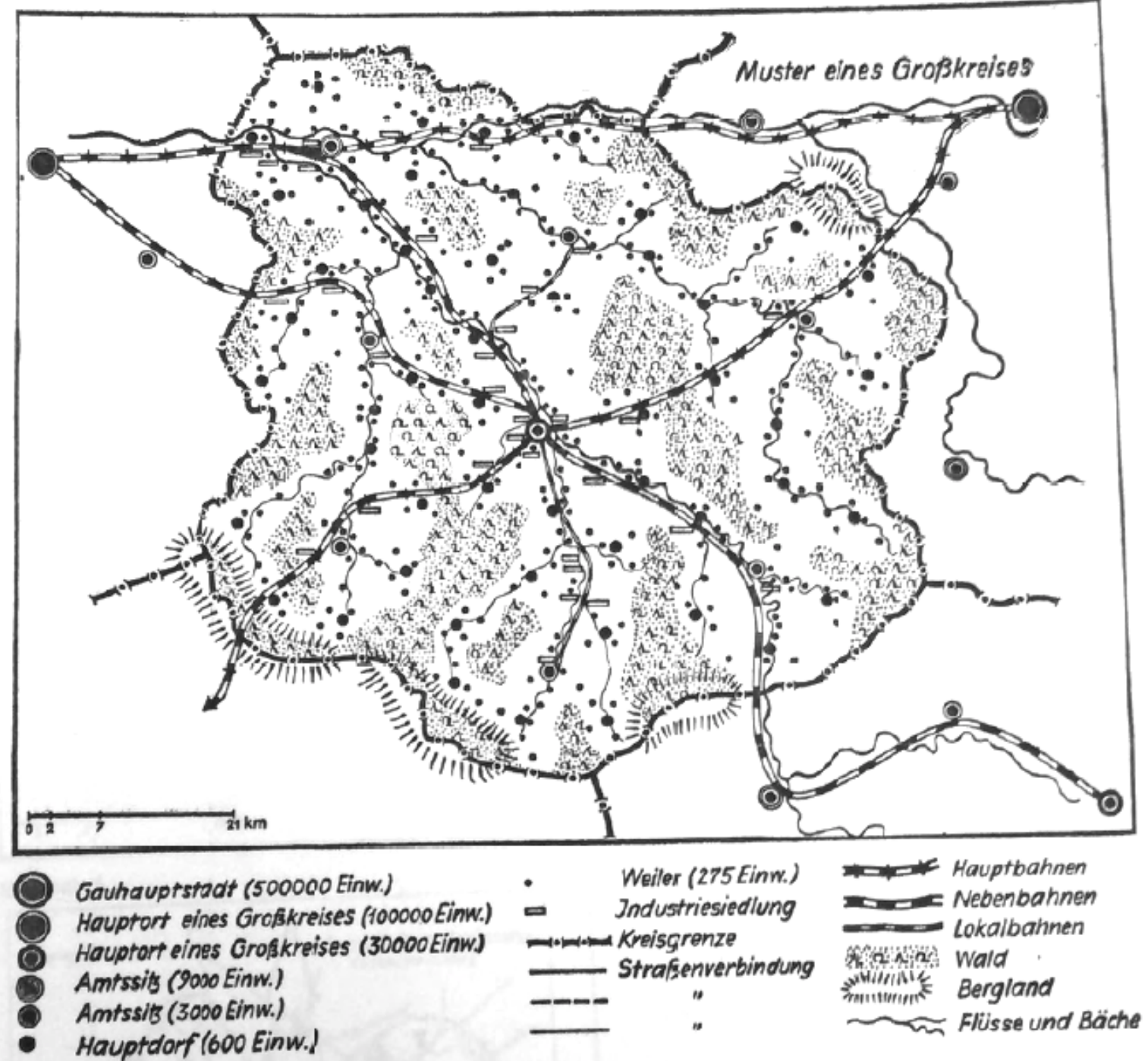

Abb. 3. Christaller (1940:310).

von Christaller, so dass sich diese beiden Arbeiten gegenseitig als empirisch und theoretisch, geographisch und wirtschaftswissenschaftlich stützten (Haggett, 1965:53; Bunge, 1962:134; Berry und Garrison, 1958; Getis, 1961:9). Zwar ist die Arbeit Löschs unter dem Schrecken von Krieg und ,the accidental way in which states are created and smashed“geschrieben und damit in gewisser Weise unter dem Eindruck der Abwesenheit räumlicher Ordnung, beiden ging es aber um die Suche nach einer ,more natural and lasting spatial order of things“ (Lösch, 1938:71). Alle der eingangs erwähnten Texte der frühen quantitativen Revolution in der anglophonen Geographie sowie zahlreiche weitere der hier genannten, reproduzieren entweder Christallers oder Löschs Abbildungen. Das Hexagon wird zu einem Logo auf Sammelbänden, Schriftenreihen und Zeitschriften, zu einem Schlagwort in den Indexen geographischer Arbeiten und zu einem pro- duktiven Instrument bei der Etablierung einer quantitativtheoretischen Geographie.

In einer englischsprachigen Publikationen erstmals Bezug genommen wird vermutlich in Ullmans ,A Theory of Location for Cities“ (Ullman, 1941). Diese Arbeit bildet einen Vorläufer zu Harris“ und Ullmanns „The Nature of Cities“ (Harris und Ullman, 1945), jenem Text, der das konzentrische Stadtmodell der Chicago School um die visuelle Repräsentation von sektoralen und multizentrischen Formationen erweitert und damit nicht zuletzt die Visualisierung von Stadtmodellen in die Stadtgeographie einbringt. Bei Ullman dient die Abbildung einer hexagonalen Struktur wie so oft als visueller Beweis dafür, dass Hexagone die optimale geometrische Figur zur Konstruktion und Abdeckung von Versorgungsgebieten seien (Abb. 5). Zudem wird von Ullman das Modell mit der Möglichkeit der Planung raumstruktureller Optima verbunden. 


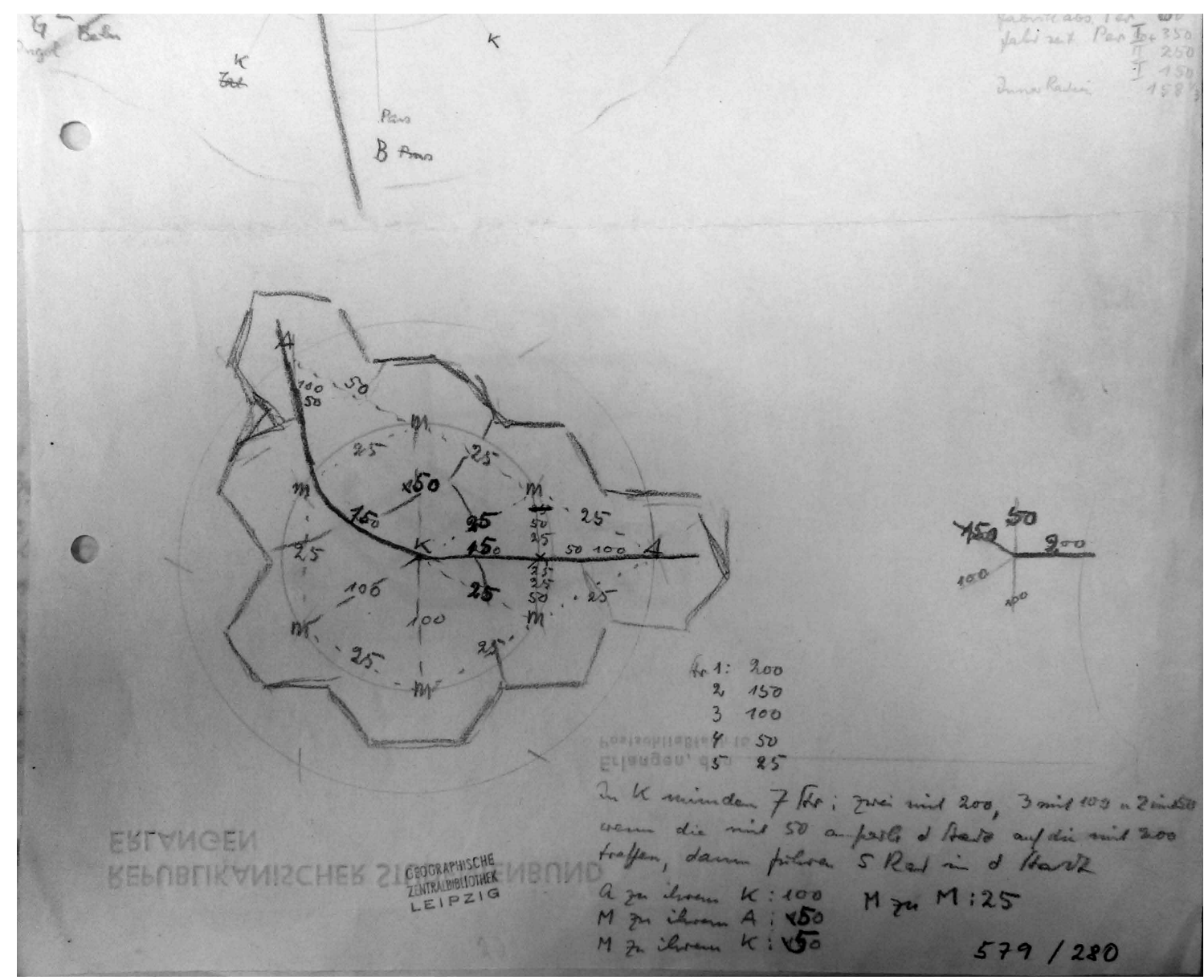

Abb. 4. NL Christaller, Institut für Länderkunde Leipzig 579/280 (Christaller, ca. 1930).

Mit der Konstituierung einer neuen Geographie - die Texte von Ullman und Harris erschienen nicht in geographischen Zeitschriften - finden sich eine wachsende Zahl von Transformationen, Übersetzungen und Versuchen, mit dem Hexagon weiterzuarbeiten, darunter Übersetzungen in andere empirische Kontexte (Berry und Garrison, 1958; Smailes, 1946; Brush, 1953), Entwürfe der Geographie als Regional Science (Isard, 1956) oder als Raumwissenschaft mit Fokus auf geometrische Muster (Bunge, 1962). Sie begegnen als einführende Diskussionen in Lehrbüchern zur Plausibilisierung des neuen Paradigmas (Haggett, 1965; Isard, 1960). Vielfach dient Christallers visuelle Repräsentation dabei als Basis für andere Visualisierungen. Sei es, dass andere Geometrien präferiert werden (Trewartha, 1943; Harris und Ullman, 1945; Hartman, 1950) oder dass die Hexagonalstruktur entlang anderer Dimensionen transformiert wird, wie es Isard bezogen auf einen nicht mehr homogen bevölkerten Raum und ausgehend von Lösch vorschlägt (Isard, 1956:272). Die Hexagonale Struktur wird hier zu etwas, mit dem man rechnen kann, etwas das nicht von der Karte oder der Theorie abgeleitet, sondern potenziell errechnet ist, etwa durch die Verschränkung räumlicher Muster mit der Bevölkerungsver- teilung in einem System (Abb. 6). Diese Übersetzung in Anamorphoten wird gerade von solchen Autoren aufgegriffen, die in den folgenden Jahren eine Mathematisierung der Kartographie für die Geographie beginnen (Tobler, 1961; Bunge, 1962; Getis, 1961). In diesen Weiterführungen, die keineswegs treu zu Christallers oder Löschs Überlegungen sind, reproduziert sich die oben diskutierte Produktivität auch auf Seiten der LeserInnen und NutzerInnen.

Und so wie Christaller Hexagone zeichnete, so findet sich auch hier anekdotische Evidenz zur Rolle des Nachsehens und Nachzeichnens. In einer Reihe von Bibliotheksbänden, auf die zum Schreiben dieses Artikels zurückgegriffen wurde, gab es mit Bleistift oder Kugelschreiber gezeichnete Hexagone, die einen Lernprozess, manchmal ein Missverständnis der Theorie und immer ein Missverständnis bezüglich Bibliothekseigentum indizieren. EinE LeserIn verband eine Reihe von 4 mal 4 Punkten, die Christaller in seiner Theorie der Zentralen Orte bei der Herleitung der hexagonalen Ordnung nutzte mit Linien um nachzuzeichnen warum eine versetzte Anordnung zentraler Orte notwendig ist (Vgl. Abb. 7). Jemand anderes erweiterte Garners Darstellung einer Hexagonalstruktur mit drei Ebenen um eine höhere und 

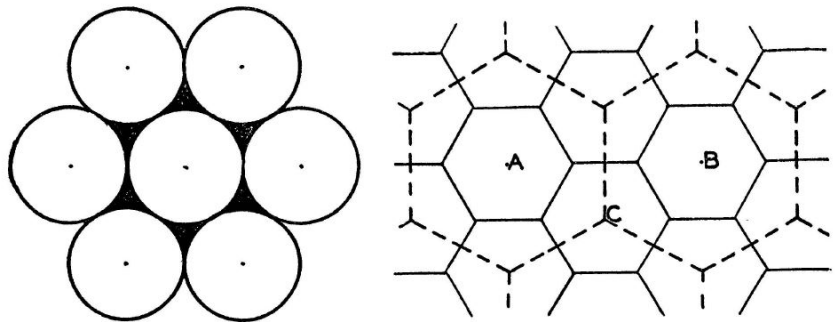

Abb. 5. Ullmann (1941:856).

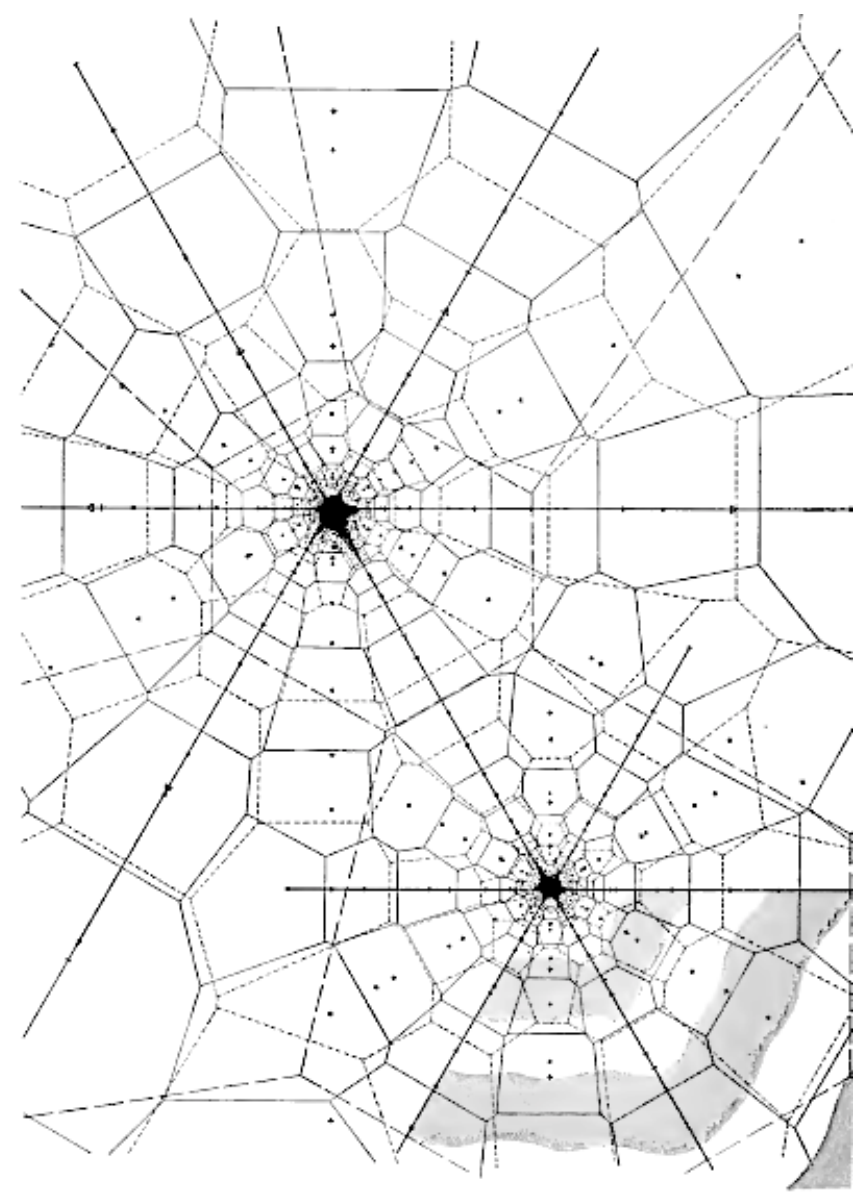

Abb. 6. Isard (1956:272).

eine niedrigere Ebene (Garner, 1967:307). Und andere versuchten alternative Hexagone in Haggetts „Locational Analysis“ (Haggett, 1965:119) und Daceys „Analysis of Central Place and Point Patterns by a Nearest Neighbor Method" (Dacey, 1962:59) zu zeichnen.

\section{Das Hexagon und das Visualitätsregime der quantitativ-theoretischen Geographie}

Der Abschließende Teil bringt die einleitende Skizze zur Visualisierungspraxis der quantitativ-theoretischen Geographie are nenuer too many nor too few places of this type pands, so that there no unsupplied part, then the there is distant from one another. This is places must be equilie at the corners of equilateral the case, however, only if the places rexagons. That is,

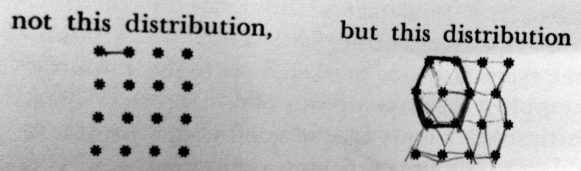

s the most uniform one. If, for instance, at a distance of $36 \mathrm{~km}$. from he central place of a certain type, central places of that same certain type hould develop, then obviously they also would be $36 \mathrm{~km}$. apart from

Abb. 7. Christaller (1966:63).

mit dieser kurzen und episodischen Rekonstruktion der Karriere von Christallers Hexagon zusammen. Zweifelsfrei erfüllt das Hexagon die wesentlichen Momente quantitativtheoretischer Visualisierung. Es ist eine diagrammatische Visualisierung eines Raumgesetzes, das Hägerstrand zu Folge erst durch diese Visualisierung intelligibel wird. Es ist vom Einzelfall abstrahiert, radikal reduktionistisch und folgt einer visuellen Sprache geometrischer Präzision und Symmetrie. Auch wurde in den vorherigen Kapiteln deutlich, wie sehr dessen Verwendung den Paradigmenwechsel der quantitativen Revolution eingebunden war und wie es als Beweis für die Tragfähigkeit dieser neuen Geographie verwendet wurde. Es stellte ebenso eine neue Form der Visualisierung dar wie auch die Visualisierung eines neuen epistemischen Dings. Es mobilisierte Verbündete aus anderen Disziplinen und hielt zugleich die Kommunikation zur alten Geographie offen.

Dies kann jedoch nicht erklären, warum es ausgerechnet Christallers Hexagon war, das eine solche Anziehungskraft entwickelte, es sei denn man sieht in ihm die erste explizit geographische Artikulation eines neuen geographischen Visualisierungsregimes, wohingegen die Darstellungen von von Thünen, Weber oder auch Lösch aus anderen Disziplinen zur Geographie sprachen. Als Aktant in der frühen quantitativ-theoretischen Geographie wurde dieses Hexagon - so die hier vertretene These - jedoch deshalb so erfolgreich, weil es viel unsauberer und vermittelnder war, als es scheint. Zwar tritt es auf in einer Ästhetik der Präzision, Rationalität, des Kalküls und dem was Porter als Rhetorik ,dünner Beschreibung“ bezeichnet (Porter, 2012), verweist auf Anforderungen nach Relevanz und Anwendung, Modellbildung und Theorie sowie auf Übersetzung und Transformation mittels mathematischer Verfahren. Es ist gereinigt von Kontingenz, Ambivalenz und lokaler Individualität, die so zentral war, für die Geographie der Länderkunde. Wenn Geometrie „the archteype of modern mind“ (Bauman, 1991:15) ist, so verkörpert es einen Modus modernistischer Visualisierung, der nach dem zweiten Weltkrieg auch in den Sozialwissenschaften an Plausibilität gewinnt (Halpern, 2014).

Aber was anhand der unterschiedlichen Nutzungen, Übersetzungen und Transformationen deutlich wurde, ist eine 
starke Flexibilität und Unschärfe. Wie Daston mit Bezug auf Porter schreibt, wurden ,,bestimmte Formen der Quantifizierung nicht deswegen mit Objektivität verbunden, weil sie notwendigerweise die Wirklichkeit genauer widerspiegeln“ (Daston, 2001:143), sondern weil sie eine wesentliche Funktion und Produktivität bei der Autorisierung und Übersetzung von Wissen ermöglichten.

Zunächst, das wurde bereits angedeutet, ist unklar, was Status und Begriff von Raum sind. Einerseits tritt das Hexagon im Modus kartographischer Rationalität auf und ist für die Geographie kartographisch lesbar. Dies gilt ganz besonders, weil das Hexagon in „Die Zentralen Orte in Süddeutschland" einhergeht mit der Veröffentlichung von Darstellungen, die eindeutig als Karten ausgewiesen sind und damit die Übersetzung zwischen Welt, Karte und Hexagon ermöglicht wird. Es ist eine räumliche Darstellung einer räumlichen Theorie. Christaller bemüht sich vielfach, auch in der abstrakten Bestimmung der hexagonalen Struktur, Distanzen und Flächen in Kilometern und Quadratkilometern zu bestimmen (Christaller, 1933a:72). Zugleich wird vom Realraum abstrahiert, spricht Christaller von ,wirtschaftlicher Entfernung“ und konstatiert, dass „,der mathematische Ausdruck der Entfernung in Meter oder Kilometern völlig nebensächlich“ sei (ebd., 31). Er plädiert dafür „einen Raum gedanklich auf[zu]bauen, einen ,reinen Raum', wie in der Physik" (Christaller, 1941b:120). Auf diese Form der relationalen und modellhaften Räumlichkeit reagieren sowohl Isard wie auch Tobler mit seinem Entwurf einer analytischen Kartographie (Tobler, 1976).

Damit wird deutlich, dass das Hexagon als ein Hybrid zwischen referenziellen und diagrammatischen Bildern funktioniert, zwischen solchen, die räumliche Arrangements abbilden und solchen, die als Diagramme Beziehungen zwischen Elementen durch Verräumlichung visualisieren. Zwar verschieben sich geographische Visualisierungen in dieser neuen Geographie im Kontinuum zwischen Tabelle-DiagrammKarte-Bild-Spiegelbild (Günzel und Nowak, 2012) in Richtung des Diagramms, handelt es sich bei diesen abstrakten geometrischen Formen und Mustern doch um logische, berechnete und rechnende Bilder, dennoch ist Hexagon weder eindeutig Karte, noch Modell, noch Diagramm. Vielmehr zirkuliert es zwischen all diesen Momenten.

Zudem changiert das Hexagon zwischen mathematischer Abstraktion und Anschaulichkeit, eine Ambivalenz, die sich durch wesentliche Teile der quantitativ-theoretischen Geographie zieht. Die Geographie bleibt trotz Hinwendung zu Theorie und Mathematik eine Disziplin des Konkreten. Der Ruf nach Mathematisierung und Raumgesetzen führte zunächst nicht dazu, dass umfangreich mit mathematischen Formeln gearbeitet wurde. Christaller wendet sich explizit gegen ein „Zuviel“ an mathematischer Präzision. Vielmehr ist es eine Geographie als Geometrie, also eine sehr visuelle und räumliche Form der Mathematik. Anders als Lösch, unternahm Christaller die Herleitung des Hexagons fern jeder mathematischen Begründung und eher kartographisch vi- suell (Christaller, 1933a:69). So heißt es in „Raumtheorie und Raumordnung“: ,Stoßen verschiedene Marktgebiete von gleichmäßig über das Land verteilten Städten unmittelbar aneinander, so wird aus dem Kreis ein Sechseck" (Christaller, 1941b:121 f.). Damit musste weder eine Mathematikausbildung nachgeholt werden, noch die kartographische Tradition über Bord geworfen werden. Einmal mehr ist es William Bunge, der dies auf den Punkt bringt, wenn er von einer ,literalness of geography“ (Bunge, 1966b:263) spricht, einer Wissenschaft, für die, William Warntz zitierend, Mengendiagramme tatsächlich auf der Erdoberfläche gezeichnet werden (ebd., 256).

\section{Schluss}

Dieser Beitrag hat einige Überlegungen zur Rolle visueller Darstellung für die frühe quantitative Revolution formuliert. Ausgangspunkt war die Beobachtung, dass es dort zu einer Vervielfältigung visueller Darstellungen kommt. Dies gilt gerade auch für theoretische Arbeiten. Von Christaller ausgehend wurde gezeigt, dass sich nicht nur Form, sondern gerade auch Funktion dieser Abbildungen massiv gewandelt hat. An die Stelle von Inventarisierung und Illustration treten nun berechnete und rechnende Bilder. Diesen wird eine erhebliche Rolle bei der Plausibilisierung eines neuen geographischen Denkens zugesprochen. Zugleich wurde hier die These vertreten, dass Christallers Hexagon sich gerade deshalb so sehr als Leitmetapher für die quantitativ-theoretische Geographie anbot, da es wesentlich unschärfer und offener war, als es auf den ersten Blick schien. Es konnte vielfältig mobilisiert werden und zugleich Zusammenhalt herstellen.

Ein neues Paradigma muss neben Texten und institutioneller Macht Instrumente bereitstellen, die eine Produktivität erzeugen. Seien es Experimentalsysteme, Maschinen oder visuelle Metaphern. Für die entstehende quantitativtheoretische Geographie gehören dazu Dinge wie der Computer und veränderte Regime universitärer Forschung und Bildung im Fordismus. Aber eben auch so banale Dinge wie ein Hexagon, eine Figur, die so omnipräsent in und für die Geographie wurde, dass selbst der erste große Angriff gegen die Dominanz einer quantitativ-theoretischen Geographie, die 1969 erschienene erste Ausgabe der Zeitschrift „Antipode", dieses im Logo trug.

Danksagung. Der Autor bedankt sich bei den TeilnehmerInnen des Workshops „Raum.Gesetze.Daten.“ in Erlangen für die Diskussion einer ersten Fassung dieses Artikels, den äußerst hilfreichen Kommentaren der GutachterInnen sowie der FritzThyssen-Stiftung für die Förderung eines Forschungsprojekts zur quantitativen Revolution in der deutschsprachigen Geographie.

Edited by: B. Korf

Reviewed by: three anonymous referees 


\section{Literatur}

Barnes, T.: Envisioning Economic Geography: Three Men and their Figures, Geogr. Z., 86, 94-105, 1998.

Barnes, T.: „Desk killers:“ Walter Christaller, central place theory, and the Nazis, in: Geographies of Knowledge and Power, Herausgeber: Meusburger, P., Gregory, D., und Suarsana, L., Dordrecht, Springer, 187-201, 2015.

Bauman, Z.: Modernity and ambivalence, Oxford, polity, ISBN-13: 0-7456-0573-7, 1991.

Berry, B. und Garrison, W.: A Note on Central Place Theory and the Range of a Good, Econ. Geogr., 34, 304-311, 1958.

Bobek, H.: Eine neue Arbeit zur Stadtgeographie: Rezension von Walter Christaller, Die zentralen Orte in Süddeutschland, Zeitschrift der Gesellschaft für Erdkunde zu Berlin, 125-130, 1935.

Brush, J.: The Hierarchy of Central Places in Southwestern Wisconsin, Geogr. Rev., 43, 380-402, 1953.

Buck-Morss, S.: Envisioning Capital: Political Economy on Display, Crit. Inquiry, 21, 434-467, 1995.

Bunge, W.: Theoretical Geography, Lund, The Royal Univ. of Lund, Gleerup, 1962.

Bunge, W.: Appendix to Theoretical Geography, Lund, C. W. K. Gleerup, 1966a.

Bunge, W.: Gerrymandering, Geography, and Grouping, Geogr. Rev., 56, 256-263, 1966b.

Burri, R. V. und Dumit, J.: Social Studies of Scientific Imaging and Visualization, in: The handbook of science and technology studies, Herausgeber: Hackett, E. J., 3. Aufl. Cambridge, MA, The MIT Press, 297-317, 2008.

Christaller, W.: Skizze, Walter Christaller Nachlass, Institut für Länderkunde, Leipzig, Box 579/28, ca. 1930.

Christaller, W.: Die Zentralen Orte in Süddeutschland, Jena, Gustav Fischer Verlag, 1933a.

Christaller, W.: Grundsätzliches zu einer Neugliederung des Deutschen Reiches und seiner Verwaltungsbezirke, Geographische Wochenschrift, 1, 913-919, 1933b.

Christaller, W.: Grundgedanken zum Siedlungs- und Verwaltungsaufbau im Osten, Neues Bauerntum, 305-312, 1940.

Christaller, W.: Die zentralen Orte in den Ostgebieten und ihre Kulutr- und Marktkbereiche, Leipzig, K. F. Koehler, 1941a.

Christaller, W.: Raumtheorie und Raumordung, Archiv für Wirtschaftsplanung, 1, 116-135, $1941 \mathrm{~b}$.

Christaller, W.: Land und Stadt in der Deutschen Volksordnung, Deutsche Agrarpolitik, 53-56, 1942.

Christaller, W.: Central Places in Southern Germany, translated by: Baskin, C. W., Englewood Cliffs, Prentice-Hall, 1966.

Christaller, W.: Wie ich zu der Theorie der zentralen Orte gekommen bin. Ein Bericht, wie eine Theorie entstehen kann, und in meinem Fall entstanden ist, Geogr. Z., 56, 88-101, 1968.

Cosgrove, D. E.: Social formation and symbolic landscape, London, Croom Helm, ISBN-13: 0-7099-0780-X, 1984.

Dacey, M. F.: Analysis of Central Place and Point Patterns by a Nearest Neighbor Method, in: Proceedings of the IGU Symposium in Urban Geography, Herausgeber: Norborg, K., , Lund, C. W. K. Gleerup, 55-75, 1962.

Daston, L. (Hrsg.): Objektivität und die Flucht aus der Perspektive, in: Wunder, Beweise und Tatsachen. Zur Geschichte der Rationalität, Frankfurt, Fischer, 127-155, 2001.
Daston, L.: Science Studies and the History of Science, Crit. Inquiry, 35, 798-813, 2009.

Daston, L.: The Humboldtian Gaze, in: Cultures and politics of research from the early modern period to the age of extremes, Herausgeber: Epple, M. und Zittel, C., Berlin, Akad.-Verl., 45-60, 2010.

Daston, L. und Galison, P.: Objectivity, New York, Zone Books, ISBN-13: 9781890951788, 2007.

Driver, F.: Visualizing geography: a journey to the heart of the discipline, Prog. Hum. Geog., 19, 123-134, 1995.

Driver, F.: Hidden histories made visible? Reflections on a geographical exhibition, T. I. Brit. Geogr., 38, 420-435, 2013.

Dyce, M.: Canada between the photograph and the map: Aerial photography, geographical vision and the state, J. Hist. Geogr., 39, 69-84, 2013.

Garner, B. J.: Models of Urban Geography and Settlement Location, in: Models in Geography, Heruasgeber: Chorley, R. und Haggett, P., London, Methuen, 303-360, 1967.

Getis, A.: A Theoretical and Empirical Inqury into the Spatial Structure of Retail Activities, Seattle, univ. Diss., University of Washington, 1961.

Godlewska, A. M. C.: From Enlightenment Vision to Modern Science? Humboldt's Visual Thinking, in: Geography and Enlightment, Herausgeber: Livingstone, D. und Withers, C., Chicago, University of Chicago Press, 236-275, 1999.

Gould, P.: Geography 1957-1977: the Augean Period, Ann. Assoc. Am. Geogr., 69, 139-151, 1979.

Gregory, D.: Geographical Imaginations, Oxford, Blackwell, ISBN10: 0-631-18331-0, 1994.

Günzel, S. and Nowak, L.: Das Medium Karte zwischen Bild und Diagramm, in: KartenWissen. Territoriale Räume zwischen Bild und Diagramm, Herausgeber: Günzel, S. und Nowak, L., Wiesbaden, Reichert, 1-32, 2012.

Hägerstrand, T.: Ansprache an Walter Christaller. Walter Christaller Nachlass, Institut für Länderkunde, Leipzig, Box 578/723-727, 24.04.1967.

Haggett, P.: Locational Analysis in Human Geography, 2. Aufl., London, Arnold, 1965.

Hagner, M.: Ansichten der WIssenschaftsgeschichte, in: Ansichten der Wissenschaftsgeschichte, Herausgeber: Hagner, M., Frankfurt, Fischer, 7-39, 2001.

Halpern, O.: Beautiful data. A history of vision and reason since 1945, Durham, NC, Duke University Press, 2014.

Haraway, D.: Situated Knowledge. The Science Question in Feminism and the Privilege of Partial Perspective, Feminist Stud., 14, 575-599, 1988.

Harris, C. und Ullman, E.: The Nature of Cities, Ann. Am. Acad. Polit. SS, 242, 7-17, 1945.

Hartman, G.: The Central Business District-A Study in Urban Geography, Econ. Geogr., 26, 237-244, 1950.

Hartshorne, R.: The Nature of Geography, A Critical Survey of Current Thought in the Light of the Past, Lancaster, Association of American Geographers, 1939.

Harvey, D.: Explanation in Geography, London, Edward Arnold, 1969.

Henniges, N.: „Sehen lernen“: Die Exkursionen des Wiener Geographischen Instituts und die Formierung der Praxiskultur der geographischen (Feld-)Beobachtung in der Ära Albrecht Penck (1885 bis 1906), Mitt. Österr. Geogr. G., 156, 141-170, 2014. 
Herf, J.: Reactionary Modernism: Technology, Culture, and Politics in Weimar and the Third Reich, Cambridge, Cambridge University Press, 1984.

Hettner, A.: Die Einheit der Geographie in Wissenschaft und Unterricht, in: Die Geographie als Wissenschaft und Lehrfach. Zehn geographische Abende im Zentralinstitut für Erziehung und Unterricht, Herausgeber: Zentralinstitut für Erziehung und Unterricht, Berlin, Ernst Siegfried Mittler und Sohn, 3-32, 1919.

Hettner, A.: Die Geographie. Breslau, Ferdinand Hirt, 1927.

Isard, W.: Location and Space-Economy. A General Theory Relating to Industrial Location, Market Areas, Land Use, Trade, and Urban Structure, New York, John Wiley \& Sons, 1956.

Isard, W.: Methods of Regional Analysis: an Introduction to Regional Science, Cambridge, MIT Press, 1960.

Jackson, P.: Maps of meaning. An introduction to cultural geography, London, Boston, Unwin Hyman, 1989.

Kegler, H.: Zentrale Orte. Transfer als „Normalisierung“, ACME: An International E-Journal for Critical Geographies, 15, 36-80, 2016.

Kegler, K. R.: Deutsche Raumplanung. Das Modell der ,zentralen Orte“ zwischen NS-Staat und Bundesrepublik, Paderborn, Schöningh, 2015.

Kuhn, T. S.: The structure of scientific revolutions, Chicago, University of Chicago Press, 1962.

Latour, B.: Visualisation and Cognition: Drawing Things Together, in: Representation in scientific practice, Herausgeber: Lynch, M. und Woolgar, S., Cambridge, Mass, MIT Press, 1-32, 1990.

Livingstone, D. N.: In defence of situated messiness: Geographical knowledge and the history of science, Geojournal, 26, 228-229, 1992.

Lösch, A.: The Nature of Economic Regions, South. Econ. J., 5, 71-78, 1938.

MacDonald, F. und Withers, C. W. J. (Hrsg.): Geography, technology and instruments of exploration. Surrey, UK England, Burlington, VT, Ashgate, 2015.

Michel, B.: Der Geographische Blick. Überlegungen zu einer Wissenschaftsgeschichte geographischer Visualitätsregime, Geogr. Z., 101, 20-35, 2013.

Michel, B.: Sehen wie ein Geograph. Ein wissenschaftsgeschichtlicher Blick darauf, wie Geographen zu Zeiten des Landschaftsparadigmas das Sehen lernten und wie dies dazu beitrug das geographische Selbst zu konstituieren, Berichte. Geographie und Landeskunde, 88, 21-38, 2014.

Michel, B.: Geographische Visualitätsregime zwischen Länderkunde und Quantitativer Revolution, in: Visuelle Geographien, Herausgeber: Schlottmann, A. und Miggelbrink, J., Bielefeld, Transcript, 209-224, 2015.

Michel, B.: „World War II was the best thing that has happened to geography“ Der Beitrag des Zweiten Weltkrieges zu einer neuen Raum- und Kartenpraxis in der Geographie, in: Medien - Krieg - Raum, Herausgeber: Nowak, L., 2016a.

Michel, B.: Seeing Spatial Structures. On the Role of Visual Material in the Making of the Early Quantitative Revolution in Geography, Geografiska Annaler B, im Erscheinen, 2016b.

Porter, T. M.: Thin Description. Surface and Depth in Science and Science Studies, Osiris, 27, 209-226, 2012.

Ratzel, F.: Anthropo-Geographie oder Grundzüge der Anwendung der Erdkunde auf die Geschichte, Stuttgart, J. Engelhorn, 1882.
Ratzel, F.: Anthropogeographie. Zweiter Teil. Die geographische Verteilung des Menschen, Stuttgart, J. Engelhorn, 1891.

Rheinberger, H.-J.: Sichtbar Machen. Visualisierung in den Naturwissenschaften, in: Bildtheorien - Anthropologische und kulturelle Grundlagen des Visualistic Turn, Herausgeber: SachsHombach, K., Frankfurt, Suhrkamp, 127-145, 2009.

Rheinberger, H.-J.: On Historicizing Epistemology, Stanford, Stanford University Press, 2010.

Rose, G.: Feminism and Geography. The Limits of Geographical Knowledge, Cambridge, polity, ISBN-10: 0-7456-0818-3, 1993.

Rose, G.: Geography as a Science of Observation: The Landscape, the Gaze and Masculinity, in: A Companion to Political Geography, Herausgeber: Agnew, J., Mitchell, K., und Toal, G., London, Blackwell, 341-350, 1996.

Rose, G.: On the Need to Ask How, Exactly, Is Geography "Visual"?, Antipode, 35, 212-221, 2003.

Rössler, M.: „Wissenschaft und Lebensraum“ Geographische Ostforschung im Nationalsozialismus: Ein Beitrag zur Disziplingeschichte der Geographie, Berlin, Reimer, 1990.

Schaefer, F. K.: Exceptionalism in Geography: A Methodological Examination, Ann. Assoc. Am. Geogr., 43, 226-249, 1953.

Schlottmann, A. und Miggelbrink, J.: Visuelle Geographien - ein Editorial, Social Geography, 4, 13-24, 2009.

Schlottmann, A. und Miggelbrink, J. (Hrsg.): Visuelle Geographien, Bielefeld, Transcript, ISBN-13: 978-3-8376-2720-6, 2015.

Schneider, B.: Linien als Reisepfade der Erkenntnis. Alexander von Humboldts Isothermenkarte des Klimas, in: KartenWissen. Territoriale Räume zwischen Bild und Diagramm, Herausgeber: Günzel, S. und Nowak, L., Wiesbaden, Reichert, 175-199, 2012.

Smailes, A.: The Urban Mesh of England and Wales, T. I. Brit. Geogr., 11, 87-101, 1946.

Stewart, J. Q.: Empirical Mathematical Rules concerning the Distribution and Equilibrium of Population, Geogr. Rev., 37, 461-485, 1947.

Tobler, W. R.: Map Transformations of Geographical Space, Seattle, univ. Diss., University of Washington, 1961.

Tobler, W. R.: Analytical Cartography, The American Cartographer, 3, 21-31, 1976.

Trewartha, G.: The Unincorporated Hamlet: One Element of the American Settlement Fabric, Ann. Assoc. Am. Geogr., 33, 3281, 1943.

Trezib, J. N.: Die Theorie der zentralen Orte in Israel und Deutschland. Zur Rezeption Walter Christallers im Kontext von Sharonplan und „Generalplan Ost“", Berlin, de Gruyter, ISBN-13: 9783-11-033813-3 , 2014.

Tschierske, H.: Raumfunktionelle Prinzipien in einer allgemeinen theoretischen Geographie: Axiomatische und empirische Bestandteile in ihr, Erdkunde, 15, 92-109, 1961.

Tuan, Y.-F.: Sight and Pictures, Geogr. Rev., 69, 413-422, 1979.

Ullman, E.: A Theory of Location for Cities, Am. J. Sociol., 46, 853-864, 1941.

von Böventer, E.: Walter Christaller's Central Places and Peripheral Areas. The Central Place Theory in Retrospect, J. Regional Sci., 9, 117-124, 1969.

Warntz, W.: Transportation, Social Physics, And The Law Of Refraction, The Professional Geographer, 9, 2-7, 1957a.

Warntz, W.: Geography of Prices and Spatial Interaction, Papers and Proceedings of the Regional Science Association, 3, 118-129, 1957b. 
Weigt, E.: Deutscher Geographentag zu Frankfurt am Main Pfingsten 1951, 12.-18. Mai 1951, Erdkunde, 5, 260-263, 1951.

Weigt, E.: Die Geographie. Eine Einführung in Wesen, Methoden, Hilfsmittel und Studium, Braunschweig, Westermann, 1957.
Wintzer, J.: Geographien erzählen. Wissenschaftliche Narrationen von Geschlecht und Raum, Stuttgart, Franz Steiner, ISBN-13: 978-3-515-10832-4, 2014. 